\title{
Homeomorphism Problems of Fuzzy Real Number Space and The Space of Bounded Functions with Same Monotonicity on $[-1,1]$
}

\author{
H.-D. Wang, S.-C. Guo, S.M. Hosseini Bamakan, Y. Shi
}

\author{
Hua-Dong Wang \\ 3. College of Mathematical Sciences, \\ Beijing, 100190, China \\ huadw2012@163.com

\section{Si-Cong Guo} \\ College of Sciences, \\ Liaoning Technical University, \\ Fuxin 123000, China
}

1. Key Laboratory of Big Data Mining and Knowledge Management,

Chinese Academy of Sciences, Beijing 100190, China

2. Research Center on Fictitious Economy \& Data Science,

Chinese Academy of Sciences, Beijing 100190, China

University of Chinese Academy of Sciences,

\section{Seyed Mojtaba Hosseini Bamakan}

1. Key Laboratory of Big Data Mining and Knowledge Management,

Chinese Academy of Sciences, Beijing 100190, China

2. Research Center on Fictitious Economy \& Data Science,

Chinese Academy of Sciences, Beijing 100190, China

3. School of Management,

University of Chinese Academy of Sciences,

Beijing, 100190, China

s_mojtabahossini@yahoo.com

\section{Yong Shi*}

1. Key Laboratory of Big Data Mining and Knowledge Management,

Chinese Academy of Sciences, Beijing 100190, China

2. Research Center on Fictitious Economy \& Data Science,

Chinese Academy of Sciences, Beijing 100190, China

*Corresponding author: yshi@ucas.ac.cn

\begin{abstract}
In this paper, based on the fuzzy structured element, we prove that there is a bijection function between the fuzzy number space $\varepsilon^{1}$ and the space $B[-1,1]$, which defined as a set of standard monotonic bounded functions with monotonicity on interval $[-1,1]$. Furthermore, a new approach based upon the monotonic bounded functions has been proposed to create fuzzy numbers and represent them by suing fuzzy structured element. In order to make two different metrics based space in $B[-1,1]$, Hausdorff metric and $L_{p}$ metric, which both are classical functional metrics, are adopted and their topological properties are discussed. In addition, by the means of introducing fuzzy functional to space $B[-1,1]$, we present two new fuzzy number's metrics. Finally, according to the proof of homeomorphism between fuzzy number space $\varepsilon^{1}$ and the space $B[-1,1]$, it's argued that not only does it give a new way to study the fuzzy analysis theory, but also makes the study of fuzzy number space easier.
\end{abstract}

Keywords: fuzzy numbers; fuzzy structured element, standard monotonic bounded functions, fuzzy functional, homeomorphism 


\section{Introduction}

Fuzzy numbers, which are a generalization of real numbers, have been perfectly applied to model and show the fuzzy data. Recently, application of fuzzy numbers in data mining algorithms has been an interesting topic to the researchers in this domain, for instance, clustering $[1,2]$, classification [3] and regression [4,5]. Generally, the efforts have been done in study of fuzzy mathematical analysis and its application falls into two main categories:

First, studies on constructing fuzzy number metrics based on the fuzzy numbers and their topological properties. Many researchers proposed different metrics and many discussions on them have been proposed. For example, Hausdorff metric [6], $L_{p}$ metric [7] and sendograph metric [8], were proposed as some of the most well-known widely used metrics.

The second category consists of those studies which addresses the relationship between the fuzzy number space and other topological spaces, study the properties of the fuzzy number space and develop some new methods in the proposed spaces. Among these studies, Goetschel and Voxman(2003) introduced a homeomorphic mapping from $\theta$-crisp fuzzy number space to Hilbert space $\ell_{2}$, which ranges in a convex cone (see [9]). Later, Gerg [10] generalized this mapping by extending the $\theta$-crisp fuzzy number space to a more general one.

In order to apply the functional analysis to the fuzzy-valued functions studies, in which variables are real numbers and function values are fuzzy numbers, Puri and Ralescu [11], proposed an embedding theorem that the fuzzy number space $\varepsilon^{1}$ can be embedded into a Banach space $X$, with the help of the Radstrom embedding theorem of compact convex set. This theorem establishes the theoretical link between the fuzzy number space and the Banach space. However, because of do not considering any specific structure of Banach space, it is not easy to implement (it is not applicable anymore). Thus, by adopting the mapping of Goetschel and Voxman, Wu and Ma [12,13] embedded fuzzy number space into the concrete Banach space $C[0,1] \times C[0,1]$ $(C[0,1]=\{f: f$ is a bounded left-continuous function on $(0,1]$, and $f$ has right limit on $(0,1]$, especially $f$ is right-continuous at 0$\}$ ), and present a specific isometrically isomorphic operator. Although the proposed embedding operator is proved to be as same as the embedding operator given by Puri and Ralescu [11] in the sense of isometrical isomorphism, the embedding operator has a specific form.

This paper is organized as follows: Section 2 and Section 3 introduced definitions and notations employed throughout the paper. In section 4, we introduced a specific fuzzy number, namely; fuzzy structured element. Then two important theorems which are the local mapping theorem and the structured element representation theorem of fuzzy number, are proved. As a result, we obtain a conclusion that there exist an one-one mapping from $B[-1,1]$ with the same order standard monotonic bounded function family on $[0,1]$ to the fuzzy real number space $\varepsilon^{1}$. In section 5, we introduced Lp metric and Hausdorff metric into $B[-1,1]$ and some of its topological properties, such as completeness and separability are discussed. In section 6 , by means of a fuzzy functional induced by fuzzy structured element, two fuzzy number metrics induced in the given metrics of $B[-1,1]$. This section discussed the homeomorphism Problems between $B[-1,1]$ and the space $\varepsilon^{1}$. Finally, we conclude and provide future works in Section 7 .

\section{Notion of the fuzzy numbers}

Fuzzy numbers are the natural generalization of real and crisp numbers. A fuzzy number is a set of the real line with the upper semi-continuous and quasi-concave membership function. The definition implies that $\alpha$-cut $\left(A_{\alpha}\right)$ of a fuzzy subset $A$ is a closed interval in $\left[A_{l}^{\alpha}, A_{r}^{\alpha}\right]$ for any $\alpha \in(0,1]$. The support of a fuzzy number $A$ is a crisp set so that $\operatorname{supp} A=\operatorname{cl}(\{x: A(x)>0\})=$ $\left[A_{l}^{0}, A_{r}^{0}\right]$ (the closure of the support of $\left.\mathrm{A}\right)$. Thus, by supposing $\operatorname{supp} A$ to be a bounded closed 
interval, $A$ defined as a bounded fuzzy number. Denote all bounded fuzzy numbers on real line $\mathbb{R}$ as $\tilde{N}_{c}(\mathbb{R})\left(\right.$ or $\left.\varepsilon^{1}\right)$.

Theorem 1. [12] If $u \in \tilde{N}_{c}(\mathbb{R})$, then let

$$
\underline{u}(\alpha)=\inf \left\{x: x \in u_{\alpha}\right\}, \quad \bar{u}(\alpha)=\sup \left\{x: x \in u_{\alpha}\right\},
$$

here $\underline{u}(\alpha)$ and $\bar{u}(\alpha)$ are two functions that satisfy the following conditions (1)-(4) on $[0,1]$ :

(1) $\underline{u}(\alpha)$ is a bounded left continuous nondecreasing function on $(0,1]$;

(2) $\bar{u}(\alpha)$ is a bounded left continuous nonincreasing function on $(0,1]$;

(3) $\underline{u}(\alpha)$ and $\bar{u}(\alpha)$ are right continuous at $\alpha=0$;

(4) $\underline{u}(\alpha) \leq \bar{u}(\alpha)$.

Conversely, if functions $\underline{u}(\alpha)$ and $\bar{u}(\alpha)$ satisfy the conditions (1)-(4) on $[0,1]$, then there exists an unique $u \in \tilde{N}_{c}(R)$ such that $u_{\alpha}=[\underline{u}(\alpha), \bar{u}(\alpha)]$ for each $\alpha \in[0,1]$.

The theorem says that for any fuzzy number $A$, it can be uniquely determined by two monotonic functions $\underline{u}(\alpha), \bar{u}(\alpha)$ on interval $[0,1]$.

\section{Notions of the Extended set-valued function and general in- verse function}

Let $f$ be a monotonic and bounded function on $[a, b]$ and $x_{0} \in(a, b)$ be a discontinuous point in $f$. By considering $f$ as a monotone increasing function, $f$ can be a surjective function from $[a, b]$ to $(-\infty,+\infty)$ by the following formula:

$$
f\left(x_{0}\right)=\left[f\left(x_{0}-\right), f\left(x_{0}+\right)\right], f(a)=(-\infty, f(a+)], f(b)=[f(b-),+\infty),
$$

Here, we denote a new function $\hat{f}$, which $\hat{f}$ is a monotonic set-valued function extended by $f$ and it also called extensional set-valued function of $f$. Furthermore, we denote all the family of function $f$ which are bounded and have the same monotonicity on $[a, b]$, by $D[a, b]$.

\subsection{Discontinue monotonic function with set-valued extensional at disconti- nuity}

For discontinue monotonic increasing function $f, x_{0}$ is a discontinuous point in the range of $[-1,1]$. Here, $f\left(x_{0}-0\right)=m_{1}$ and $f\left(x_{0}+0\right)=m_{2}$, by considering our default suppose that $f$ is an increasing function, then $m_{1}<m_{2}$ and $f\left(x_{0}\right)$ is an interval number between $\left[m_{1}, m_{2}\right]$. If functional values of all discontinuities redefined as closed interval with left-hand and righthand limited values, then this new function is called monotonic bounded set-valued function extensional from $f$ that we denote it by $\hat{f}$. Obviously, inverse function $\hat{f}^{-1}$ of $\hat{f}$ exist.

\subsection{Continuous non-strictly monotonic function}

Suppose $f$ is a non-strictly increasing function, then there exists at least one pair points $\left\{x_{1}, x_{2}\right\}$ on $[-1.1]$ such that value of $f$ is equal to constant $c=f\left(x_{1}\right)=f\left(x_{2}\right)$ on interval $\left[x_{1}, x_{2}\right]$. And suppose $x_{1}, x_{2}$ are two endpoints so that increasing function $f$ is equal to constant, that is, when $x<x_{1}, f(x)<c$ and when $x>x_{2}, f(x)>c$. Here, we define inverse function $f^{-1}(x)$ which is continue close to 0 at discontinuity, i.e. when $x_{2} \leq 0, \hat{f}^{-1}(c)=\lim _{x \rightarrow c^{+0}} f^{-1}(x)=y_{+}$; 
when $x_{1} \geq 0, \hat{f}^{-1}(c)=\lim _{x \rightarrow c^{-0}} f^{-1}(x)=y_{-}$; when $0 \in\left[x_{1}, x_{2}\right]$, define $f^{-1}(c)$ as set of two points $\left\{y_{-}, y_{+}\right\}$, denote by $\hat{f}^{-1}(c)=\left\{y_{-}, y_{+}\right\}$.

It is quite straightforward to verify that, if $f$ is an increasing and bounded function on $[-1,1]$ and $\hat{f}$ is the extensional set-valued function of $f$, then the inverse function of $\hat{f}$ can be equivalently defined as:

$$
\hat{f}^{-1}(x)=\left\{\begin{array}{lc}
\sup \{t: \hat{f}(t)=x,-1 \leqslant t<0\}, & -\infty<x \leqslant f(0-) \\
0, & f(0-) \leqslant x \leqslant f(0+) . \\
\inf \{t: \hat{f}(t)=x, 0<t \leqslant 1\}, & f(0+) \leqslant x<+\infty
\end{array}\right.
$$

Example 2. To make the above concept more understandable, let consider $f$ as a monotonic bounded function on $[0,2]$,

$$
f(x)=\left\{\begin{array}{ll}
x, & 0 \leqslant x \leqslant 1 \\
1+x, & 1<x \leqslant 2
\end{array} .\right.
$$

Then its extensional set-valued function $\hat{f}(x)$ and its inverse function $\hat{f}^{-1}(x)$ are defined as the following:

$$
\hat{f}(x)=\left\{\begin{array}{ll}
x, & 0 \leqslant x<1 \\
{[1,2],} & x=1 \\
1+x, & 1<x \leqslant 2
\end{array} \quad, \quad \hat{f}^{-1}(x)=\left\{\begin{array}{ll}
x, & 0 \leqslant x<1 \\
1, & 1 \leqslant x<2 \\
x-1, & 2 \leqslant x \leqslant 3
\end{array} .\right.\right.
$$

The $f(x)$ and $\hat{f}(x)$ can be illustrated by Figure 1
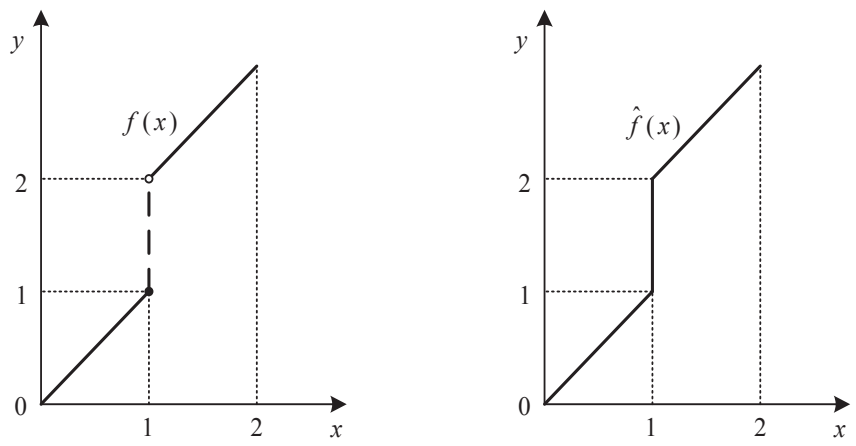

Figure 1: Set-valued function $\hat{f}$ extended by $f$

\section{$4 \quad$ Fuzzy structured element and transformation}

In order to establish the relationship between the fuzzy real number space $\varepsilon^{1}$ and the monotone function space on interval $[-1,1]$, we introduce a method, namely the Fuzzy Structured Element, which was proposed by Sicong Guo in [14].

Definition 3. Let $E$ be a fuzzy set on real line $\mathbb{R}$ and $E(x)$ is membership function of $E$. Then, $E$ is called a fuzzy structured element, if $E(x)$ satisfies the following properties:

1) $E(0)=1$;

2) $E(x)$ is monotonic increasing and right-continuous on $[-1,0)$, monotonic decreasing and leftcontinuous on $(0,1]$; 
3) For any $x \in(-\infty,-1) \cup(1,+\infty), E(x)=0$.

Further, $E$ is called a normal fuzzy structured element if the fuzzy structured element $E$ satisfies: (1) $E(x)>0$ for all $x \in(-1,1)$; (2) $E(x)$ is continuous, strictly monotonic increasing on $[-1,0)$ and also continuous, strictly monotonic decreasing on $(0,1]$.

According to Definition 3, it is easy to know that the fuzzy structured element is a special fuzzy number on real line $\mathbb{R}$, which can be used to express the concept of fuzzy zero $\tilde{0}$.

Let $E$ be a fuzzy number. $E$ is called a triangular structured element if it has membership function $\mu_{E}(x)$, where

$$
\mu_{E}(x)=\left\{\begin{array}{lc}
1-x, & x \in[0,1] \\
1+x, & x \in[-1,0] \\
0, & \text { otherwise }
\end{array}\right.
$$

As it is shown in Figure 2. Obviously, $E$ is a special fuzzy structured element.

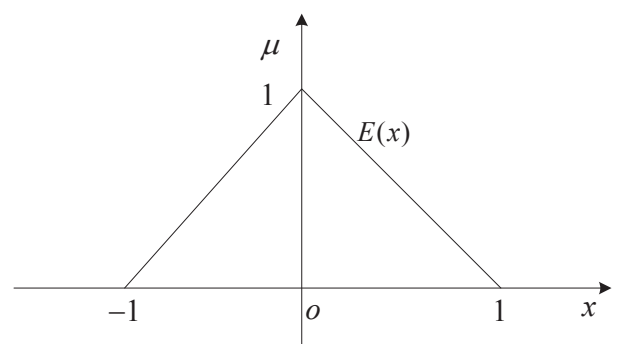

Figure 2: Triangular structured element $E$

Based on the fuzzy structured element, we can give the following two theorems:

Theorem 4 (Local Mapping Theorem). Suppose $E$ is a fuzzy structured element on $\mathbb{R}$ with membership function $E(x) . f(x)$ is monotonically bounded on $[-1,1]$ and $\hat{f}(x)$ is extensional setvalued function of $f(x)$. Then $\hat{f}(E)$ is a bounded closed fuzzy number and membership function of $\hat{f}(E)$ is $E\left(\hat{f}^{-1}(x)\right)$, where $\hat{f}^{-1}(x)$ is the inverse function of $\hat{f}(x)$

(If $f(x)$ is strictly increasing and continuous on $[-1,1]$, then $\hat{f}^{-1}(x)$ is a ordinary inverse function of $f(x))$.

Proof: Let $A=\hat{f}(E), A(y)$ defined as a membership function of $\hat{f}(E)$. Suppose that $f(x)$ is increasing and bounded on $[-1,1]$. By extension principle, we have

$$
A(y)=\bigvee_{y \in \hat{f}(x)} E(x), \hat{f}(E)=\bigcup_{x \in \mathbb{R}} E(x) * \hat{f}(x),
$$

where

$$
E(x) * \hat{f}(x)(y)=\left\{\begin{array}{ll}
E(x), & y \in \hat{f}(x) \\
0, & \text { otherwise }
\end{array} .\right.
$$

From the former equation, the membership function of $\hat{f}(E)$ is $\hat{f}(E)(y)$. When $y \in \hat{f}(x)$, counterpart membership degree defined as $E(x)$.

Denote $\alpha$-cut of $E$ by $E_{\alpha}=\left[e_{\alpha}^{-}, e_{\alpha}^{+}\right]$. It follows from the concept of fuzzy structured element that $E_{0}=\left[e_{0}^{-}, e_{0}^{+}\right] \subseteq[-1,1]$. Since $f(x)$ is increasing bounded on interval $[-1,1]$ and $\hat{f}(x)$ is surjection on $\mathbb{R}$, it follows that for $\alpha \in(0,1]$,

$$
[\hat{f}(E)]_{\alpha}=\hat{f}\left(E_{\alpha}\right)=\hat{f}\left[e_{\alpha}^{-}, e_{\alpha}^{+}\right]=\left[f\left(e_{\alpha}^{-}\right), f\left(e_{\alpha}^{+}\right)\right],
$$


For $\alpha=0$,

$$
[\hat{f}(E)]_{0}=\overline{\operatorname{supp} \hat{f}(E)}=\overline{\cup_{\alpha \in(0,1]}[\hat{f}(E)]_{\alpha}}=\overline{\cup_{\alpha \in(0,1]}\left[\hat{f}\left(E_{\alpha}\right)\right]}=\left[f\left(e_{0}^{-}+\right), f\left(e_{0}^{+}-\right)\right]
$$

Thus $E_{\alpha}, \alpha \in[0,1]$ are bounded closed sets.

For all $\alpha_{1}, \alpha_{2} \in(0,1]$, if $\alpha_{1} \leq \alpha_{2}$, then $E_{\alpha_{1}} \subseteq E_{\alpha_{2}}$, that is $e_{\alpha_{2}}^{-} \leq e_{\alpha_{1}}^{-}, e_{\alpha_{1}}^{+} \leq e_{\alpha_{2}}^{+}$. Since $f(x)$ is monotone increasing, we have

$$
f\left(e_{\alpha_{1}}^{-}-\right) \leq f\left(e_{\alpha_{2}}^{-}-\right), f\left(e_{\alpha_{2}}^{+}+\right) \leq f\left(e_{\alpha_{1}}^{+}+\right)
$$

Therefore, $[\hat{f}(E)]_{\alpha_{1}} \subseteq[\hat{f}(E)]_{\alpha_{2}}$. Furthermore, it follows from Eq.(3) that

$$
[\hat{f}(E)]_{0}=\overline{\cup_{\alpha \in(0,1]}[\hat{f}(E)]_{\alpha}} \supseteq[\hat{f}(E)]_{\alpha_{1}},
$$

It means that $\hat{f}(E)$ is a convex set on real line $\mathbb{R}$.

Since $E$ is a fuzzy number and 1-cut set $E_{1}$ of $E$ is nonempty and $[\hat{f}(E)]_{1}=\hat{f}\left(E_{1}\right)$ also is nonempty, hence we can say that $\hat{f}(E)$ is a normal fuzzy number. From definition of bounded closed fuzzy number, we know $\hat{f}(E) \in \tilde{N}_{c}(R)$.

Since $\hat{f}(E)(y)=\vee_{y \in \hat{f}(x)} E(x)=E\left(x_{y}\right)$ as $y \in \hat{f}(x)$, where $x_{y}=\hat{f}^{-1}(y)$. It follows that

$$
\hat{f}(E)(y)=E\left(\hat{f}^{-1}(y)\right)
$$

or

$$
\hat{f}(E)(x)=E\left(\hat{f}^{-1}(x)\right) .
$$

If $f(x)$ is monotonic decreasing on $[-1,1]$, the proof can be shown in a similar manner.

Theorem 5 (Theorem of Structured Element Expression of Fuzzy Number ). For a given regular fuzzy structured element $E$ and any bounded fuzzy number $A$, there exists a monotonic bounded function $f$ on $[-1,1]$ such that $A=f(E)$ (strictly, exists a extended set-valued function $\hat{f}$ such that $A=\hat{f}(E)$ ). We called it fuzzy number A generated by the fuzzy structured element.

Proof: From fuzzy number expression theorem, fuzzy number $u$ can be expressed by a family set $\left\{u_{\alpha}: u_{\alpha}=[\underline{u}(\alpha), \bar{u}(\alpha)], \alpha \in[0,1]\right\}$. Therefore, we just need to prove that there exists a monotone bounded function $f(x)$ on $[-1,1]$ such that $f(E)=u$, that is, for all $\alpha \in[0,1]$, $[f(E)]_{\alpha}=[\underline{u}(\alpha), \bar{u}(\alpha)]$.

Let

$$
f(x)=\left\{\begin{array}{ll}
\underline{u}(E(x)), & x \in[-1,0] \\
\bar{u}(E(x)), & x \in(0,1]
\end{array} .\right.
$$

It follows from Theorem 1 that $f(x)$ is a monotone increasing bounded function on $[-1,1]$. From the local mapping principle, $f(E)$ is a bounded closed fuzzy number. It follows from the extension principle that $[f(E)]_{\alpha}=f\left(E_{\alpha}\right)$. Denote $E(x)$ on $[-1,0]$ as $l_{E}(x)$ and $E(x)$ on $[0,1]$ as $r_{E}(x)$. Since $E$ is strictly increasing on $[-1,0]$ and is also a bijection from $[-1,0]$ to $[0,1], E$ is strictly decreasing on $[0,1]$ and is also a bijection from $[0,1]$ to $[0,1]$, so $l_{E}(x), r_{E}(x)$ inverse and are denoted by $l_{E}^{-1}(\alpha), r_{E}^{-1}(\alpha)$, then

$$
E_{\alpha}=[\underline{E}(\alpha), \bar{E}(\alpha)]=\left[l_{E}^{-1}(\alpha), r_{E}^{-1}(\alpha)\right] .
$$

Since $\underline{u}(\alpha), \bar{u}(\alpha)$ are left-continuous on $(-1,0]$ and are right-continuous at $\alpha=0$, also $\mathrm{E}(\mathrm{x})$ is continuous, we know that $\underline{u}(E(x))=\underline{u}\left(l_{E}(x)\right)$ is left-continuous on $(-1,0]$ and is right-continuous at $x=-1, \bar{u}(E(x))=\bar{u}\left(r_{E}(x)\right)$ is right-continuous on $[0,1)$ and is left-continuous at $x=1$. Since 
$f(x)$ is increasing, it follows that

for all $\alpha \in(0,1]$,

$$
\begin{aligned}
& f\left(E_{\alpha}\right)=f\left[l_{E}^{-1}(\alpha), r_{E}^{-1}(\alpha)\right]=\left[f\left(l_{E}^{-1}(\alpha)-\right), f\left(r_{E}^{-1}(\alpha)+\right)\right] \\
& =\left[\underline{u}\left(E\left(l_{E}^{-1}(\alpha)+\right)\right), \bar{u}\left(E\left(r_{E}^{-1}(\alpha)-\right)\right)\right] \\
& =\left[\underline{u}\left(E\left(l_{E}^{-1}(\alpha)\right)\right), \bar{u}\left(E\left(r_{E}^{-1}(\alpha)\right)\right)\right] \\
& =[\underline{u}(\alpha), \bar{u}(\alpha)]
\end{aligned}
$$

for $\alpha=0$,

$$
\begin{aligned}
& f\left(E_{0}\right)=f\left[l_{E}^{-1}(0), r_{E}^{-1}(0)\right]=[f(-1+), f(1-)] \\
& =[\underline{u}(E(-1+)), \bar{u}(E(1-))] \\
& =[\underline{u}(E(-1)), \bar{u}(E(1))] \\
& =[\underline{u}(0), \bar{u}(0)]
\end{aligned}
$$

Therefore, we conclude that $[f(E)]_{\alpha}=[\underline{u}(\alpha), \bar{u}(\alpha)]$ for all $\alpha \in[0,1]$. That's to say that $f(E)=u$. We complete the proof of this theorem.

When no confusion can arise in the following discussions, we will use $f(x)$ to denote the extended function $\hat{f}(x)$ and use $f(E)$ to instead of $\hat{f}(E)$, respectively.

Theorem 6. Let $f$ be a monotonic bounded function and $E$ be a fuzzy structured element on $\mathbb{R}$ and fuzzy number $u=f(E)$. For all $\alpha \in[0,1], E_{\alpha}=\left[e_{\alpha}^{-}, e_{\alpha}^{+}\right]$. Then

(1) If $f(x)$ is increasing on $[-1,1]$, then $\alpha$-cut of fuzzy number $u$ is closed interval

$$
u_{\alpha}=\left\{\begin{array}{ll}
{\left[f\left(e_{\alpha}^{-}-\right), f\left(e_{\alpha}^{+}+\right)\right],} & \alpha \in(0,1], \\
{\left[f\left(e_{\alpha}^{-}+\right), f\left(e_{\alpha}^{+}-\right)\right],} & \alpha=0,
\end{array} .\right.
$$

(2) If $f(x)$ is monotonic decreasing function on $[-1,1]$, then $\alpha$-cut of $u$ is closed interval

$$
u_{\alpha}= \begin{cases}{\left[f\left(e_{\alpha}^{+}-\right), f\left(e_{\alpha}^{-}+\right)\right],} & \alpha \in(0,1], \\ {\left[f\left(e_{\alpha}^{+}+\right), f\left(e_{\alpha}^{-}-\right)\right],} & \alpha=0,\end{cases}
$$

Proof: Based on Theorem 4 and function $f$ satisfies the monotone condition of Local Mapping Theorem, it follows that for any $\alpha \in(0,1]$, we have $[f(E)]_{\alpha}=f\left(E_{\alpha}\right)$. Since $f$ is monotone on closed interval $E_{\alpha}=\left[e_{\alpha}^{-}, e_{\alpha}^{+}\right] \subseteq[-1,1]$, it follows that:

If $f$ is increasing, for $\alpha \in(0,1]$, we have

$$
\begin{aligned}
u_{\alpha} & =f\left(E_{\alpha}\right)=f\left[e_{\alpha}^{-}, e_{\alpha}^{+}\right] \\
& =\left[\inf \left\{y: y \in f\left[e_{\alpha}^{-}, e_{\alpha}^{+}\right]\right\}, \sup \left\{y: y \in f\left[e_{\alpha}^{-}, e_{\alpha}^{+}\right]\right\}\right] \\
& =\left[f\left(e_{\alpha}^{-}-\right), f\left(e_{\alpha}^{+}+\right)\right],
\end{aligned}
$$

and for $\alpha=0$, it holds that

$$
\begin{aligned}
u_{0} & =\overline{\operatorname{supp} f(E)}=\overline{f(E)_{\dot{0}}}=\overline{f\left(E_{\dot{0}}\right)} \\
& =\overline{\varlimsup_{\alpha \rightarrow 0}\left[f\left(e_{\alpha}^{-}-\right), f\left(e_{\alpha}^{+}+\right)\right]}=\left[f\left(e_{0}^{-}+\right), f\left(e_{0}^{+}-\right)\right] .
\end{aligned}
$$


If $f$ is decreasing, we have

$$
\begin{aligned}
u_{\alpha} & =f\left(E_{\alpha}\right)=f\left[e_{\alpha}^{-}, e_{\alpha}^{+}\right] \\
& =\left[\inf \left\{y: y \in f\left[e_{\alpha}^{-}, e_{\alpha}^{+}\right]\right\}, \sup \left\{y: y \in f\left[e_{\alpha}^{-}, e_{\alpha}^{+}\right]\right\}\right] \\
& =\left[f\left(e_{\alpha}^{+}+\right), f\left(e_{\alpha}^{-}-\right)\right],
\end{aligned}
$$

From the Local Mapping Theorem 4, we know that given a fuzzy structured element $E$, it will be transformed into an fuzzy number $A=f(E)$ with any a monotonic function $f$ on $[-1,1]$. When $f$ is not a monotonic function, the fuzzy set $f(A)$ can not be guaranteed to be a fuzzy number. Theorem 5 show us that for any bounded fuzzy number $A$, we always can find a monotonic bounded function $f$ on $[-1,1]$ such that $f(E)=A$. Therefore, the two theorems reveal to us that there exists a deep relationship between the family of bounded monotonic function on $[-1,1]$ and the fuzzy number space.

\section{The same order standard monotonic bounded function classes $B[-1,1]$} have

Let $f$ be monotonic bounded function on $[-1,1]$. If for any discontinuity $x$ in $[-1,1]$, we

$$
f(x)=\frac{1}{2}[f(x+)+f(x-)],
$$

where $f(x+)(f(x-))$ is the right-limit(left-limit) of $f(x)$ at the point $x$, then $f(x)$ is called a standard monotonic bounded function on $[-1,1]$. All same order standard monotonic bounded function on $[-1,1]$ is denoted by $B[-1,1]$.

It is obvious that a continuous monotonic bounded function on $D[-1,1]$ is also a standard monotonic bounded function.

Definition 7. Suppose that $f \in D[-1,1]$, we define

$$
\check{f}(x)= \begin{cases}f(-1+), & x=-1 \\ {[f(x-)+f(x+)] / 2,} & x \in(-1,1) \\ f(1-), & x=1\end{cases}
$$

where $\check{f}(x)$ is as a standardized form of $f(x)$. Obviously, $\check{f} \in B[-1,1]$. If $f$ is a standard monotonic bounded function, then $\check{f}=f$.

In the following we introduced two distance formulas:

$$
\begin{aligned}
& d_{p}(f, g)=\left[\int_{-1}^{1}|f(x)-g(x)|^{p} \mathrm{~d} x\right]^{1 / p}, \text { for all } f, g \in B[-1,1], \\
& d_{H}(f, g)=\sup _{x \in[-1,1]}|f(x)-g(x)|, \text { for all } f, g \in B[-1,1] .
\end{aligned}
$$

where $1 \leq p<+\infty$.

Theorem 8. Let $E$ be a normal fuzzy structured element, $K$ is a bounded closed interval on $R$. Denote

$$
B_{f}(K)=\{f: f \in B[-1,1] \text { and }[f(-1), f(1)] \subseteq K\},
$$

Metric spaces $\left(B[-1,1], d_{H}\right)$ and $\left(B_{f}(K), d_{p}\right)$ both are complete. 
Proof: First, we prove completeness of space $\left(B[-1,1], d_{p}\right)$. Suppose each elements in $\left(B[-1,1], d_{p}\right)$ are increasing, for decreasing situation has similarity conclusions. Suppose given sequence $\left\{x_{n}(t)\right\}$, where $x_{n}(t) \in\left(B[-1,1], d_{p}\right), n=1,2, \cdots$.

Let $d_{p}\left(x_{n}, x_{m}\right) \rightarrow 0$, (as $\left.n, m \rightarrow \infty\right)$, that is, sequence $\left\{x_{n}(t)\right\}$ satisfies Cauchy uniformly convergence conditions. Suppose $x_{0}(t)$ is limit of sequence $x_{n}(t)$,i.e.

$$
\lim _{n \rightarrow \infty} x_{n}(t)=x_{0}(t) \text { for all } t \in[-1,1] .
$$

Since all $x_{n}\left(t_{1}\right)$ is monotonic increasing function, then $x_{n}\left(t_{1}\right) \leq x_{n}\left(t_{2}\right)$ for all $t_{1}, t_{2} \in[-1,1]$. So

$$
\lim _{n \rightarrow \infty} x_{n}\left(t_{1}\right) \leq \lim _{n \rightarrow \infty} x_{n}\left(t_{2}\right),
$$

and $x_{0}\left(t_{1}\right) \leq x_{0}\left(t_{2}\right)$. Thus $x_{0}(t)$ is monotonic increasing function.

Now we prove $g(t)$ is standard function on $[-1,1]$. Suppose $t_{0}$ is a discontinuous point of $g(t)$. We might as well suppose that $g(t)$ is't standard, that is

$$
g\left(t_{0}\right) \neq \frac{1}{2}\left[g\left(t_{0}+\right)+g\left(t_{0}-\right)\right] .
$$

Let $\delta=\left|g\left(t_{0}\right)-\frac{1}{2}\left[g\left(t_{0}+\right)+g\left(t_{0}-\right)\right]\right|$. Furthermore, since $f_{n}(t), n=1,2, \cdots$, are standard, we have

$$
\begin{aligned}
& E\left(t_{0}+\right)\left|f_{n}\left(t_{0}+\right)-g\left(t_{0}+\right)\right|<\varepsilon, \\
& E\left(t_{0}-\right)\left|f_{n}\left(t_{0}-\right)-g\left(t_{0}-\right)\right|<\varepsilon, \\
& E\left(t_{0}\right)\left|f_{n}\left(t_{0}\right)-g\left(t_{0}\right)\right|<\varepsilon .
\end{aligned}
$$

Take $\varepsilon=\varepsilon_{0}<\delta / 2$. When $n \geq n_{0}\left(\varepsilon_{0}\right)$, we have

$$
\begin{aligned}
& \left|g\left(t_{0}\right)-\frac{1}{2}\left[g\left(t_{0}+\right)+g\left(t_{0}-\right)\right]\right| \\
& \leq\left|f_{n}\left(t_{0}\right)-g\left(t_{0}\right)\right|+\frac{1}{2}\left|f_{n}\left(t_{0}+\right)-g\left(t_{0}+\right)\right|+\frac{1}{2}\left|f_{n}\left(t_{0}-\right)-g\left(t_{0}-\right)\right| \\
& \leq \varepsilon_{0}+\varepsilon_{0}=2 \varepsilon_{0}<\delta
\end{aligned}
$$

a contradiction. Thus, $g(t)$ is standard on $[-1,1]$.

2) Now we prove the metric space $\left(B_{f}(K), d_{p}\right)$ is complete. Suppose that $f_{n}$ is a Cauchy sequence in $B_{f}(K), d_{p}$, then for any $\varepsilon>0$, there exists a positive integer $N$ such that for any $m, n>N$, we have

$$
d_{H}\left(f_{m}, f_{n}\right)=\left[\int_{-1}^{1}\left|f_{m}(t)-f_{n}(t)\right|^{p} \mathrm{~d} x\right]^{1 / p}<\varepsilon .
$$

This indicates that $\left\{f_{n}\right\}$ is a Cauchy sequence of $L_{p}[-1,1]$. We know that $L_{p}[-1,1]$ is complete space, so $\left\{f_{n}\right\}$ is converse in $L_{p}[-1,1]$. Suppose $h$ is a limit of sequence $\left\{f_{n}\right\}$. Similar to the proof in 1) that $h(x)$ is increasing and bounded in interval $[-1,1]$. Therefore, $h(-1+)$ and $h(1-)$ exist. Let

$$
f(x)= \begin{cases}h(-1+), & x=-1 \\ {[h(x+)+h(x-)] / 2,} & x \in(-1,1) . \\ h(1-), & x=1\end{cases}
$$

It is obvious that $f(x) \in B[-1,1]$ and also is a limit of Cauchy sequence $f_{n} . f(x)$ and $h(x)$ have different values which happened only on discontinuity, so we have $d_{p}\left(f_{n}, f\right)=d_{p}\left(f_{n}, g\right)$. Thus, $f$ is a limit of Cauchy sequence in $\left(B_{f}(K), d_{p}\right)$, that is, $\left\{f_{n}\right\}$ converges in $\left(B_{f}(K), d_{p}\right)$. Hereby, the completeness of $\left(B_{f}(K), d_{p}\right)$ have been proved. 
Theorem 9. Let $E$ be a normal fuzzy structured element and $K \in I(\mathbb{R})$ be a nonempty set, denoted by:

$$
B_{f}(K)=\{f: f \in B[-1,1] \text { and }[f(-1), f(1)] \subseteq K\},
$$

then the metric space $\left(B_{f}(K), d_{p}\right)$ is complete.

Example 10. The metric space $\left(B[-1,1], d_{p}\right)$ is not complete. For example, $f_{n} \in B[-1,1]$ is defined by

$$
f_{n+1}(x)=\left\{\begin{array}{ll}
f_{n}(x), & x \in\left[-1,1-1 / n^{2}\right] \\
n, & x \in\left(1-1 / n^{2}, 1\right]
\end{array},(n \geq 1),\right.
$$

where $f_{1}(x)=0, x \in[-1,1]$. It is obvious that $f_{n}(x)(n \geq 1)$ are bounded functions. Suppose $m \leq n$, we have

$$
\begin{aligned}
d_{p}\left(f_{m}, f_{n}\right) & =\left[\int_{-1}^{1}\left|f_{m}(t)-f_{n}(t)\right|^{p} \mathrm{~d} t\right]^{1 / p}<\left[\frac{1}{(n+1)^{2}}+\frac{1}{(n+2)^{2}}+\cdots+\frac{1}{m^{2}}\right]^{1 / p} \\
& <\left[\frac{1}{n}-\frac{1}{m}\right]^{1 / p}<\frac{1}{n} \rightarrow 0(m, n \rightarrow \infty)
\end{aligned}
$$

Thus, $\left\{f_{n}\right\}$ is a Cauchy Sequence, their standard function sequence $\left\{\check{f}_{n}(x)\right\}$ is a Cauchy Sequence in $B[-1,1]$. It is easy to understand that $\left\{\check{f}_{n}(x)\right\}$ do not convergence to any upper bounded function.

Theorem 11. Metric space $\left(B[-1,1], d_{H}\right)$ is not a separable space and metric space $\left(B[-1,1], d_{p}\right)$ is a separable space.

Proof: 1) It is sufficient to construct an uncountable set $B_{f}$ of $B[-1,1]$ with the property that if $f, g \in B_{f}$, then $d_{H}(f, g)=1$. For each $t \in(-1,1)$, define $f_{t} \in B[-1,1]$ by

$$
f_{t}(x)=\left\{\begin{array}{ll}
0 & x \in[-1, t) \\
0.5 & x=t \\
1 & x \in(t, 1]
\end{array} .\right.
$$

Denote $B_{f}=\left\{f_{t}: t \in(-1,1)\right\}$. Consequently, if $t_{1} \neq t_{2}$, then $d_{H}\left(f_{t_{1}}, f_{t_{2}}\right)=1$.

2) Since $L_{p}[-1,1]$ is a separable space with respect to the metric $d_{p}$ and $B[-1,1] \subset L_{p}[-1,1]$, then $B[-1,1]$ is separable with respect to metric $d_{p}$ (For any separable metric space $X$, any nonempty subset of this space is also separable).

In general, $f \in B[-1,1],-f \in B[-1,1]$ unless $f$ is a constant-valued function. Because, if $f$ isn't constant-valued function, despite $-f$ is also monotonic function, but it is not same order with $f$. Hence, $B[-1,1]$ cannot form group with respect to operation of addition,just can form a semigroup.

It should be noted that each element in $B[-1,1]$ is not a closed form with respect to ordinary subtraction operator. We can take an example, function obtain by two monotonic function subtracted may be non-monotonic. Therefore, $B[-1,1]$ can't form linear space with respect to addition and number multiply operation.

Theorem 12. $B[-1,1]$ is a convex cone with 0 as its vertex.

The theorem is obvious, so the proof is omitted. 


\section{Topological relationship between $B[-1,1]$ and $\tilde{N}_{c}(\mathbb{R})$}

\subsection{Two types of fuzzy number metric spaces induced by the fuzzy structured element}

Let $E$ be a symmetrical regular fuzzy structured element on real line $\mathbb{R}$ and $\tilde{N}_{c}(\mathbb{R})$ be the set of all bounded closed fuzzy numbers. For given function $f \in B[-1,1]$, there exists corresponding unique fuzzy number such that $A_{f}=f(E)$. In other words, fuzzy structured element determines a mapping from $B[-1,1]$ to $\tilde{N}_{c}(\mathbb{R})$.

Denote

$$
\begin{aligned}
H_{E}: B[-1,1] & \rightarrow \tilde{N}_{c}(\mathbb{R}), \\
f & \rightarrow H_{E}(f)=f(E) \in \tilde{N}_{c}(\mathbb{R}) .
\end{aligned}
$$

Then $H_{E}$ is called fuzzy functional induced by fuzzy structured element $E$.

Using metrics $d_{p}$ and $d_{H}$ on $B[-1,1]$, mapping $H_{E}$ induces distances

$$
\begin{gathered}
d_{N p}(A, B)=d_{p}\left(H_{E}^{-1}(A), H_{E}^{-1}(B)\right), \\
d_{N H}(A, B)=d_{H}\left(H_{E}^{-1}(A), H_{E}^{-1}(B)\right),
\end{gathered}
$$

on $\tilde{N}_{c}(\mathbb{R})$, where $H_{E}^{-1}(A), H_{E}^{-1}(B)$ are preimage of mapping $H_{E}$ at $A$ and $B$, respectively. Suppose $A=f(E), B=f(E)$, where $f, g \in B[-1,1]$, then Eq.(11) and Eq.(12) can also rewrite as

$$
\begin{gathered}
d_{p}(f, g)=d_{p}\left(H_{E}(f), H_{E}(g)\right), \\
d_{H}(f, g)=d_{H}\left(H_{E}(f), H_{E}(g)\right),
\end{gathered}
$$

$\left(\tilde{N}_{c}(\mathbb{R}), d_{N p}\right)$ and $\left(\tilde{N}_{c}(\mathbb{R}), d_{N H}\right)$ are said to be distance space induced by $\left(B[-1,1], d_{p}\right)$ and $\left(B[-1,1], d_{H}\right)$, respectively. It is easy to understand that $H_{E}$ is an isometric bijection of $B[-1,1]$ onto $\tilde{N}_{c}(\mathbb{R})$.

Using isometric bijection $H_{E}$, we can translate metric of elements in fuzzy number space to metric between the same order standard monotonic bounded functions in range of $[-1,1]$. Then, what is the relationship between those metrics and the other metrics on fuzzy numbers?

Before discussing the relationship, a Lemma need to be presented here:

For $u \in \tilde{N}_{c}(\mathbb{R}), E$ is a normal fuzzy structured element. If $g \in B[-1,1]$ such that $u=g(E)$, as defined in the following:

$$
g_{u}(x)=\left\{\begin{array}{cc}
\underline{u}(E(x)) & -1 \leq x \leq 0 \\
\bar{u}(E(x)) & 0<x \leq 1
\end{array} .\right.
$$

Lemma 13. Suppose that $E$ is a normal fuzzy structured element, fuzzy number $u \in \tilde{N}_{c}(\mathbb{R}), u_{\alpha}=$ $[\underline{u}(\alpha), \bar{u}(\alpha)](\alpha \in[0,1])$. If $u=f(E), f \in B[-1,1]$, then

$$
f(x)=\check{g}_{u}(x), x \in[-1,1],
$$

where $\check{g}_{u}$ is the standard function of $g_{u}$.

\section{Proof:}

The proof has been provided in the following two steps: 
1) First, we prove $\check{g}_{u}=u$. According to the decomposition theorem, we only need to prove that $\left[\check{g}_{u}(E)\right]_{\alpha}=u_{\alpha}$ for any $\alpha \in(0,1]$. Denote $E_{\alpha}=\left[e_{\alpha}^{-}, e_{\alpha}^{+}\right]$. From the extension principle and $g(x)$ is a increasing function, it follows that

$$
\left[\check{g}_{u}(E)\right]_{\alpha}=\check{g}_{u}\left(E_{\alpha}\right)=\check{g}_{u}\left(\left[e_{\alpha}^{-}, e_{\alpha}^{+}\right]\right)=\left[\check{g}_{u}\left(e_{\alpha}^{-}-\right), \check{g}_{u}\left(e_{\alpha}^{+}+\right)\right] .
$$

Since $\underline{u}(\alpha), \bar{u}(\alpha)$ are continuous on $(0,1]$, we have $\underline{u}(E(x))$ is left-continuous on $(-1,0]$ and $\bar{u}(E(x))$ is right-continuous on $[0,1)$. Therefore,

$$
\begin{aligned}
& {\left[\check{g}_{u}\left(e_{\alpha}^{-}-\right), \check{g}_{u}\left(e_{\alpha}^{+}+\right)\right]=\left[g_{u}\left(e_{\alpha}^{-}-\right), g_{u}\left(e_{\alpha}^{+}+\right)\right] } \\
= & {\left[\underline{u}\left(E\left(e_{\alpha}^{-}\right)\right), \bar{u}\left(E\left(e_{\alpha}^{+}\right)\right)\right]=[\underline{u}(\alpha), \bar{u}(\alpha)] }
\end{aligned}
$$

That is, $\left[\check{g}_{u}(E)\right]_{\alpha}=u_{\alpha}$. Thus, $\check{g}_{u}=u$.

2) Here we prove $f$ is unique in $B[-1,1]$. Suppose $f_{1}, f_{2} \in B[-1,1]$ such that $\hat{f}_{1}(E)=$ $\hat{f}_{2}(E)=u$, then $\hat{f}_{1}^{-1}=\hat{f}_{2}^{-1}$. Furthermore, we have $\hat{f}_{1}=\hat{f}_{2}$, then $f_{1}=f_{2}$. Thus, $f$ is unique in $B[-1,1]$. Therefore, $f(x)=\check{g}_{u}(x), x \in[-1,1]$. The proof is complete.

The following theorem shows the relation between the induced fuzzy number metrics $d_{N H}, d_{N p}$ and the previous metrics of fuzzy numbers.

Theorem 14. Let $E$ be regular structured element, $u, v \in \tilde{N}_{c}(\mathbb{R})$, there are $f, g \in B[-1,1]$ such that $u=f(E), v=g(E)$. Denote $u_{\alpha}=[\underline{u}(\alpha), \bar{u}(\alpha)], v_{\alpha}=[\underline{v}(\alpha), \bar{v}(\alpha)]$, then

$$
\begin{aligned}
d_{N p}(u, v) & =\left[\int_{-1}^{1}|f(x)-g(x)|^{p} \mathrm{~d} x\right]^{1 / p} \\
& =\left[\int_{0}^{1}|\underline{u}(\alpha)-\underline{v}(\alpha)|^{p} \mathrm{~d} \underline{E}(\alpha)+|\bar{u}(\alpha)-\bar{v}(\alpha)|^{p} \mathrm{~d} \bar{E}(\alpha)\right]^{1 / p} \\
d_{N H}(u, v) & =\sup _{x \in[-1,1]}|f(x)-g(x)|=\sup _{x \in[-1,1]}(|\underline{u}(\alpha)-\underline{v}(\alpha)| \vee|\bar{u}(\alpha)-\bar{v}(\alpha)|)
\end{aligned}
$$

Proof: 1) We have from Lemma 13 that

$$
d_{N p}(u, v)=d_{p}(f, g)=d_{p}\left(\check{f}_{u}, \check{g}_{v}\right),
$$

And $\check{g}_{u}(x)=g_{u}(x)$ and $\check{g}_{v}(x)=g_{v}(x)$ are bounded almost everywhere on $[-1,1]$ respectively, it follows that $d_{p}\left(\check{f}_{u}, \check{g}_{v}\right)=d_{p}\left(f_{u}, g_{v}\right)$. Therefore,

$$
\begin{aligned}
& d_{N p}(u, v)=d_{p}\left(f_{u}, g_{v}\right)=\left[\int_{-1}^{1}\left|f_{u}(x)-g_{v}(x)\right|^{p} \mathrm{~d} x\right]^{1 / p} \\
& =\left[\int_{-1}^{0}|\underline{u}(E(x))-\underline{v}(E(x))|^{p} \mathrm{~d} x+\int_{0}^{1}|\bar{u}(E(x))-\bar{v}(E(x))|^{p} \mathrm{~d} x\right]^{1 / p}
\end{aligned}
$$

Denote $E(x)=l_{E}(x)$ for $x \in[-1,0]$ and $r_{E}(x)=E(x)$ for $x \in[0,1]$. Since $E$ is a regular fuzzy structured element, we know that $l_{E}$ is bijective from $[-1,0]$ to $[0,1], r_{E}$ is bijective from $[0,1]$ to $[0,1]$. Thus, we can say that $l_{E}^{-1}, r_{E}^{-1}$ exist and they are monotone bijections. It obvious that $\underline{E}=l_{E}^{-1}$ and $\bar{E}=r_{E}^{-1}$ both are differentiable almost everywhere. Therefore, we have

$$
\begin{aligned}
d_{N p}(u, v) & =\left[\int_{0}^{1}|\underline{u}(\alpha)-\underline{v}(\alpha)|^{p} \mathrm{~d} l_{E}^{-1}(\alpha)+\int_{1}^{0}|\bar{u}(\alpha)-\bar{v}(\alpha)|^{p} \mathrm{~d} r_{E}^{-1}(\alpha)\right]^{1 / p} \\
& =\left[\int_{0}^{1}|\underline{u}(\alpha)-\underline{v}(\alpha)|^{p} \mathrm{~d} \underline{E}(\alpha)-\int_{0}^{1}|\bar{u}(\alpha)-\bar{v}(\alpha)|^{p} \mathrm{~d} \bar{E}(\alpha)\right]^{1 / p}
\end{aligned}
$$


2) From Lemma 13, we obtain

$$
\left.d_{N H}(u, v)=d_{H}(f, g)=d_{H} \check{f}_{u}, \check{g}_{v}\right),
$$

Furthermore, $f_{u}(x), g_{u}(x)$ both are left-continuous on $(-1,0]$ and right-continuous on $[0,1)$, and are right-continuous at $x=-1$ and left-continuous at $x=1$, we have

$$
\begin{aligned}
\left|f_{u}(x)-g_{v}(x)\right| & \leq\left|f_{u}(x-)-g_{v}(x-)\right| \vee\left|f_{u}(x+)-g_{v}(x+)\right|, \quad x \in(-1,1) \\
\left|f_{u}(x)-g_{v}(x)\right| & =\left|f_{u}(x)-g_{v}(x)\right|, \quad x \in\{-1,1\}
\end{aligned}
$$

Since $\check{f}_{u}(x), \check{g}_{u}(x)$ are standard functions of $f_{u}(x), g_{v}(x)$ for $x \in(-1,1)$, we have

$$
\begin{aligned}
\left|\check{f}_{u}(x-)-\check{g}_{v}(x-)\right| & =\left|f_{u}(x-)-g_{v}(x-)\right| ; \\
\left|\check{f}_{u}(x+)-\check{g}_{v}(x+)\right| & =\left|f_{u}(x+)-g_{v}(x+)\right| .
\end{aligned}
$$

It follows that $d_{H}\left(\check{f}_{u}, \check{g}_{v}\right)=d_{H}\left(f_{u}, g_{v}\right)$. Moreover, we have

$$
\begin{aligned}
d_{H}\left(f_{u}, g_{v}\right) & =\sup _{x \in[-1,1]}\left\{\left|f_{u}(x)-g_{v}(x)\right|\right\} \\
& =\sup _{x \in[-1,0]}\{|\underline{u}(E(x))-\underline{v}(E(x))|\} \vee \sup _{x \in[0,1]}\{|\bar{u}(E(x))-\bar{v}(E(x))|\}
\end{aligned}
$$

Let $\alpha=E(x)$ on $[-1,0]$ and $[0,1]$, respectively. Since $E$ is a regular fuzzy structured element, it follows that

$$
\begin{aligned}
& \sup _{x \in[-1,0]}\{|\underline{u}(E(x))-\underline{v}(E(x))|\}=\sup _{\alpha \in[0,1]}\{|\underline{u}(\alpha)-\underline{v}(\alpha)|\}, \\
& \sup _{x \in[0,1]}\{|\bar{u}(E(x))-\bar{v}(E(x))|\}=\sup _{\alpha \in[0,1]}\{|\bar{u}(\alpha)-\bar{v}(\alpha)|\},
\end{aligned}
$$

completing the proof of the theorem.

Since integral variables $\underline{E}(\alpha), \bar{E}(\alpha)$ in the Eq.(6.1) are two general functions, the definite integral is a Riemann-Stieltjes integral. When the $E$ is a triangular structured element (2), we have $\mathrm{d} \underline{E}(\alpha)=\mathrm{d}(\alpha-1)=\mathrm{d} \alpha, \mathrm{d} \bar{E}(\alpha)=\mathrm{d}(-\alpha+1)=-\mathrm{d} \alpha$. Then the Eq.(2) becomes the following form:

$$
d_{N p}(u, v)=\left[\int_{0}^{1}|\underline{u}(\alpha)-\underline{v}(\alpha)|^{p} \mathrm{~d} \alpha-|\bar{u}(\alpha)-\bar{v}(\alpha)|^{p} \mathrm{~d} \alpha\right]^{1 / p} .
$$

From Eq.(16) in the Theorem 14, we also note that the induced fuzzy number metric $d_{N H}(u, v)$ is the same as the Hausdorff metric(Diamond,1989) [6].

\subsection{Homeomorphism between the fuzzy number space $\tilde{N}_{c}(\mathbb{R})$ and $B[-1,1]$}

Proposition 15. Suppose that $\left(X, d_{X}\right),\left(Y, d_{Y}\right)$ are two metric spaces. $F$ is an isometric bijection from $\left(X, d_{X}\right)$ to $\left(Y, d_{Y}\right)$. Then $F$ is continuous and inverse mapping $F^{-1}$ of $F$ exists and is also continuous. 
Proof: Since $F$ is a bijection of $\left(X, d_{X}\right)$ into $\left(Y, d_{Y}\right)$, there exists inverse mapping $F^{-1}$ which is also one-to-one mapping. By definition of continuous mapping, for all $x_{0} \in X$ and any positive number $\varepsilon$, there always exists a positive number $\delta$ such that $d_{Y}\left(F(x), F\left(x_{0}\right)\right)<\varepsilon$ as $d_{X}\left(x, x_{0}\right)<\delta$. Since $d_{X}\left(x, x_{0}\right)=d_{Y}\left(F(x), F\left(x_{0}\right)\right.$ ), given $\varepsilon$, it is sufficient by taking $\delta \leq \varepsilon$ (For instance, take $\delta=\varepsilon / 2$ ). Hence, $F$ is continuous. Similarly, we can also prove inverse mapping $F^{-1}$ which is also continuous.

Given a bounded closed interval $K$, let the uniformly bounded fuzzy number set as

$$
\tilde{N}_{c}(K)=\left\{u: u \in \tilde{N}_{c}(\mathbb{R}) \text { and } \operatorname{supp} u \subseteq K\right\},
$$

Since there exists a bijection $H_{E}$ of $B[-1,1]$ into $\tilde{N}_{c}(\mathbb{R})$ and $H_{E}$ and inverse function $H_{E}^{-1}$ are continuous, thus we have conclusions as follows:

Theorem 16. Metric spaces $\left(B[-1,1], d_{H}\right)$ and $\left(\tilde{N}_{c}(\mathbb{R}), d_{N H}\right)$ are homeomorphic. Metric spaces $\left(B_{f}(K), d_{p}\right)$ and $\left.\left(\tilde{N}_{c}(K)\right), d_{N p}\right)$ are homeomorphic.

Since space $\left(\tilde{N}_{c}(\mathbb{R}), d_{N H}\right)$ and $\left(B[-1,1], d_{H}\right)$ are homeomorphic, that is, both metric spaces are topologically equivalent. So elements in both of them have consistent properties on metrics. There are one-to-one relationship between fuzzy number sequence $\left\{u_{n}\right\}$ of $\left(\tilde{N}_{c}(\mathbb{R}), d_{N H}\right)$ and function sequence $\left\{f_{n}\right\}$, fuzzy number sequence on $\left(\tilde{N}_{c}(\mathbb{R}), d_{N H}\right)$ and function sequence on $\left(B[-1,1], d_{H}\right)$ have completely same properties. Similarly, fuzzy number sequence on $\left(\tilde{N}_{c}(K), d_{N p}\right)$ and function sequence on $\left(B_{f}(K), d_{H}\right)$ have completely same properties. Therefore, the properties of convergence sequence of general metric spaces are also founded to the convergence fuzzy number sequence. Thus, they are trivial to the following corollaries.

Corollary 17. Fuzzy number metric space $\left(\tilde{N}_{c}(\mathbb{R}), d_{N H}\right)$ is complete and $\left(\tilde{N}_{c}(\mathbb{R}), d_{N p}\right)$ is not complete.

Corollary 18. For any nonempty closed interval $K$ on $\mathbb{R},\left(\tilde{N}_{c}(K), d_{N p}\right)$ is a complete metric space.

Corollary 19. For any fuzzy number sequence $\left\{u_{n}\right\}$ of fuzzy number space $\left(\tilde{N}_{c}(\mathbb{R}), d_{N}\right)$ only has a limit almost everywhere. That is, the limit of convergence sequence is unique.(Where $d_{N}$ represents $d_{N H}$ or $\left.d_{N p}\right)$.

Corollary 20. Suppose that $\left\{u_{n}\right\}$ is a fuzzy number sequence of $\left(\tilde{N}_{c}(\mathbb{R}), d_{N H}\right),\left\{f_{n}\right\}$ is a function sequence on $\left(B[-1,1], d_{H}\right)$. For all $n \geq 1, u_{n}=f_{n}(E)$. Then fuzzy number sequence $\left\{u_{n}\right\}$ converge if and only if function sequence $\left\{f_{n}\right\}$ converge. Let

$$
\lim _{n \rightarrow \infty} u_{n}=u_{0}, \lim _{n \rightarrow \infty} f_{n}=f_{0},
$$

then $u_{0}=f_{0}(E)$.

If using $d_{N p}, d_{p}, B_{f}(K)$ (defined as 10) instead of $d_{N H}, d_{H}$ and $B[-1,1]$ in Corollary 20, respectively. The Corollary is still founded.

\section{Conclusion}

By using monotonic mapping of the fuzzy structured element, we have proved that the bounded fuzzy number space is homeomorphic to the space $B[-1,1]$ of monotonic bounded function with same monotonicity on $[-1,1]$. Therefore, the problem of the fuzzy number space can be transformed to one's of space $B[-1,1]$, such as the convergence of sequence of fuzzy numbers, the convergence of the fuzzy series, continuous of fuzzy-valued function and so on. To some extent, our study provides a new way for the study of fuzzy analysis. 


\section{Acknowledgements}

This work has been partially supported by grants from National Natural Science Foundation of China (Grant No.71331005, No.71110107026).

\section{Bibliography}

[1] M.-S. Yang, C.-H. Ko (1996), On a class of fuzzy c-numbers clustering procedures for fuzzy data, Fuzzy sets and systems,84(1):49-60.

[2] U. Reuter(2011), A fuzzy approach for modeling non-stochastic heterogeneous data in engineering based on cluster analysis, Integrated Computer-Aided Engineering,18(3):281-289.

[3] A. Li, Y. Shi, J. He, Y. Zhang (2011), A fuzzy linear programming-based classification method, International Journal of Information Technology \& Decision Making, 10(06):11611174 .

[4] K. Lin, P. Pai, Y. Lu, P. Chang (2013), Revenue forecasting using a least-squares support vector regression model in a fuzzy environment, Information Sciences, 220:196-209.

[5] H. Wang, S. Guo, L. Yue (2014), An approach to fuzzy multiple linear regression model based on the structural element theory, Systems Engineering - Theory 85 Practice, 34(10): 26-28.

[6] P. Diamond, P. Kloeden (1989), Characterization of compact subsets of fuzzy sets, Fuzzy Sets and Systems, 29(3):341-348.

[7] P. Diamond, P. Kloeden (1990), Metric spaces of fuzzy sets, Fuzzy sets and systems, 35(2): 241-249.

[8] P. Diamond, P. E. Kloeden, P. E. Kloeden (1994), A. Mathematician, P. E. Kloeden, Metric spaces of fuzzy sets: theory and applications, World Scientific.

[9] R. Goetschel, W. Voxman (1983), Topological properties of fuzzy numbers, Fuzzy Sets and Systems, 10(1):87-99.

[10] L. Gerg (1992), Generalisation of the goetschel-voxman embedding, Fuzzy sets and systems, 47(1): 105-108.

[11] M. L. Puri, D. A. Ralescu (1983), Differentials of fuzzy functions, Journal of Mathematical Analysis and Applications, 91(2):552-558.

[12] C.X. Wu, M. Ma (1991), Embedding problem of fuzzy number space: Part i, Fuzzy Sets and Systems, 44(1):33-38.

[13] C.X. Wu, M. Ma(1992), Embedding problem of fuzzy number space: part ii, Fuzzy Sets and Systems, 45(2):189-202.

[14] S. Guo(2002), Method of structuring element in fuzzy analysis, Journal of Liaoning Technical University, 21(5):670-673. 